\title{
BMJ Open Costing the impact of interventions during pregnancy in the UK: a systematic review of economic evaluations
}

\author{
Sophie Relph (D) , ${ }^{1}$ Louisa Delaney, ${ }^{1}$ Alexandra Melaugh, ${ }^{2}$ Matias C Vieira (D) , ${ }^{1,3}$ \\ Jane Sandall, ${ }^{1}$ Asma Khalil, ${ }^{4,5}$ Dharmintra Pasupathy, ${ }^{1,6}$ Andy Healey, ${ }^{7}$ On behalf of \\ DESiGN Trial Team
}

To cite: Relph S, Delaney L, Melaugh A, et al. Costing the impact of interventions during pregnancy in the UK: a systematic review of economic evaluations. BMJ Open 2020;10:e040022. doi:10.1136/ bmjopen-2020-040022

- Prepublication history and additional material for this paper is available online. To view these files, please visit the journal online (http://dx.doi.org/10. 1136/bmjopen-2020-040022).

Received 05 May 2020 Revised 21 September 2020 Accepted 01 0ctober 2020

Check for updates

(c) Author(s) (or their employer(s)) 2020. Re-use permitted under CC BY-NC. No commercial re-use. See rights and permissions. Published by BMJ.

For numbered affiliations see end of article.

Correspondence to

Dr Sophie Relph;

sophie.relph@kcl.ac.uk

\section{ABSTRACT}

Objective The aim of this review was to summarise the current evidence on the costing of resource use within UK maternity care, in order to facilitate the estimation of incremental resource and cost impacts potentially attributable to maternity care interventions.

Methods A systematic review of economic evaluations was conducted by searching Medline, the Health Management Information Consortium, the National Health Service (NHS) Economic Evaluations Database, CINAHL and National Institute for Health and Care Excellence (NICE) guidelines for economic evaluations within UK maternity care, published between January 2010 and August 2019 in the English language. Unit costs for healthcare activities provided to women within the antenatal, intrapartum and postnatal period were inflated to 2018-2019 prices. Assessment of study quality was performed using the Quality of Health Economic Analyses checklist.

Results 0 5084 titles or full texts screened, 37 papers were included in the final review (27 primary research articles, 7 review articles and 3 economic evaluations from NICE guidelines). Of the 27 primary research articles, 21 were scored as high quality, 3 as medium quality and 3 were low quality. Variation was noted in cost estimates for healthcare activities throughout the maternity care pathway: for midwife-led outpatient appointment, the range was £27.34-£146.25 (mean £81.78), emergency caesarean section, range was £1056.44-£4982.21 (mean $£ 3508.93$ ) and postnatal admission, range was $£ 103.00-$ $£ 870.10$ per day (mean £469.55).

Conclusions Wide variation exists in costs applied to maternity healthcare activities, resulting in challenges in attributing cost to maternity activities. The level of variation in cost calculations is likely to reflect the uncertainty within the system and must be dealt with by conducting sensitivity analyses. Nationally agreed prices for granular unit costs are needed to standardise cost-effectiveness evaluations of new interventions within maternity care, to be used either for research purposes or decisions regarding national intervention uptake.

PROSPERO registration number CRD42019145309.

\section{Strengths and limitations of this study}

- This systematic review is the first to compare published unit costs for the different healthcare activities that may be offered to women in the UK during antenatal, intrapartum and postnatal care.

- A comprehensive search was conducted in four databases; unit costs extracted from published research articles were compared with one another and with summary costs published as part of review articles (mostly national Health Technology Assessments) and guidelines.

- The search was limited to reports published since January 2010 (to ensure the data was contemporaneous) and studies conducted in the UK.

- Unit costs were extracted for common or presumed high-cost activities within the maternity pathway, where the frequency of use was expected to vary with implementation of new antenatal or intrapartum interventions; low-cost interventions, for which the frequency of use was not expected to vary, were not costed.

- Unit costs extracted from published reports of economic evaluations were inflated to 2018/2019 prices and stratified by quoted cost perspective to ensure comparability.

\section{BACKGROUND}

Healthcare economic evaluations are pertinent components of healthcare research and quality improvement, informing policymakers on the cost-effectiveness of new interventions and thereby assisting in decisions regarding their uptake. The UK National Institute for Health and Care Excellence (NICE) bases its recommendations on the implementation of new interventions according to both their clinical efficacy and cost-effectiveness. ${ }^{1}$ The number of costeffectiveness evaluations published annually in obstetrics and gynaecology has increased 
since 2000, with the majority being conducted alongside a clinical trial. ${ }^{2}$

Internationally, maternity care is funded using different payment models, including itemised bills and payment using composite 'bundled' costs. ${ }^{3-5}$ Bundled pricing describes a model where a single price is used to cover a full package of care for a specific indication. Bundled costs can be uplifted locally using factors that account for geographical variation in the cost of providing care and by the level of comorbidity or complexity of the woman and her pregnancy, but are not explicitly changed by differences in utilisation of maternity services. ${ }^{46}$ Such models are often used because they are easier for hospitals to manage, allow flexibility within the pathway and are intended to encourage improvements in care including standardisation of evidencebased care. ${ }^{3}$ Bundled payments were introduced by the Medicaid initiative (USA) to also reduce interventions that are not medically indicated and potentially harmful. ${ }^{5}$ Bundled payment models are also used for non-maternity indications. ${ }^{7}$

While bundled costs represent the cost of a woman's care to a commissioner or insurer, to the hospital they only reflect the average cost of women who experience the same level of complexity in pregnancy. Bundled payments present difficulties in estimating small changes to the overall cost of a woman's maternity care as a result of a new intervention, because the cost of her care is estimated as a composite, which is often not affected by small changes in resource use (eg, additional antenatal appointments or ultrasound scans). This represents a significant limitation for using these tariffs in estimating costs within economic evaluations, which seek to identify the true clinical resource impact of initiatives aimed at improving quality of maternal and perinatal care, as distinct from potential financial impacts to commissioners or insurers.

The aim of this review was to summarise the current evidence on the costing of resource use within UK maternity care, in order to facilitate the estimation of incremental resource and cost impacts potentially attributable to maternity care interventions.

\section{METHODS}

This review was registered on PROSPERO during the data collection stage. The report has been written using the Preferred Reporting Items for Systematic Reviews and Meta-Analyses guidelines; the checklist has been included in online supplemental file 1 .

\section{Search strategy}

A systematic review was conducted in August 2019 of the Medline, Health Management Information Consortium, the National Health Service (NHS) Economic Evaluations Database, CINAHL database and National Institute for Health and Care Excellence (NICE) guidelines for economic evaluations of maternity care and the economic impact of research interventions. Search terms included free text and expanded synonyms for terms relevant to economic evaluations (eg, cost-effectiveness and price tariffs) and to pregnancy healthcare (eg, midwife, maternity and pregnancy). The full search strategy is included within online supplemental file 2. The search was limited to papers written in the English language (because the perspective was that of UK maternity care providers) and published since 2010 (to ensure the cost estimations were recent and more reliable when inflated to current prices).

For inclusion in the review, it was predetermined that papers must be full reports of primary research studies or systematic reviews (including those in NICE guidelines), where an economic evaluation of an antenatal or intrapartum intervention was performed and assessed within the UK context only. A UK context was chosen because it is well-established internationally that different countries vary in their approach to providing maternity care, the type of clinical resource inputs used to deliver specific types of clinical activity and in terms of the efficiency with which this is delivered. A review on the international economics of childbirth identified no accepted cost that was translatable across international settings ${ }^{8}$ due to differences in national clinical practices, outcome definitions and healthcare funding mechanisms. It was also necessary that the papers reported unit costs for at least one of the maternity pathway activities listed in table 1 .

\begin{tabular}{|c|c|c|}
\hline Antenatal activity & Intrapartum activity & Postnatal/neonatal activity \\
\hline $\begin{array}{l}\text { Midwife-led antenatal } \\
\text { appointment. } \\
\text { Obstetrician-led antenatal } \\
\text { appointment. } \\
\text { Glucose tolerance test. } \\
\text { Attendance to day } \\
\text { assessment unit/triage. } \\
\text { Antenatal inpatient admission. } \\
\text { Sonography-led ultrasound } \\
\text { scan. } \\
\text { Consultant-led ultrasound } \\
\text { scan. }\end{array}$ & $\begin{array}{l}\text { Induction of labour. } \\
\text { Augmentation of labour. } \\
\text { Epidural. } \\
\text { Normal vaginal birth. } \\
\text { Instrumental vaginal birth. } \\
\text { Elective caesarean section. } \\
\text { Emergency caesarean section. } \\
\text { Repair third/fourth degree tear. } \\
\text { Manual removal of placenta. } \\
\text { Treatment of postpartum haemorrhage }(500-1500 \mathrm{~mL}) . \\
\text { Treatment of major obstetric haemorrhage }(>1500 \mathrm{~mL}) . \\
\text { Examination under anaesthesia for haemorrhage. }\end{array}$ & $\begin{array}{l}\text { Maternal stay in postnatal } \\
\text { ward (with/without baby). } \\
\text { Maternal stay in high- } \\
\text { dependency unit. } \\
\text { Maternal stay in intensive } \\
\text { care unit. }\end{array}$ \\
\hline
\end{tabular}


This list contains common and presumed high-cost maternity activities for which the frequency of use may be affected by interventions introduced in research settings; that is, activities that were expected to impact upon cost estimates calculated in economic evaluations of maternity care. There were no specific exclusion criteria. The titles and abstracts were screened by the lead author (SR), and the remaining full texts were reviewed in full against the inclusion criteria by two authors (SR and LD).

\section{Data extraction}

Data were extracted from each paper by two authors (SR and LD) onto a prespecified study spreadsheet on the cost perspective taken by the study, year and methodology used for costing the resource use. Cost perspective refers to the level at which the costs are assessed; the most common examples are patients and families, a single healthcare provider, the health and/or social services and wider society. ${ }^{9}$ Unit costs quoted for any of the key activities listed in table 1 were collected. Costs were inflated to the 2018/2019 financial year using the Department of Health's Pay \& Price Series for financial years 2008/20092015/2016 and the NHS Improvement Economic Assumptions for years 2016/2017 to 2018/2019. ${ }^{10} 11$

\section{Assessment of study quality}

Assessment of study quality was performed using the Quality of Health Economic Analyses (QHES) checklist. ${ }^{12}$ This was designed by health economists and validated by both clinicians and economists with the aim of providing a tool suitable to evaluate all common types of health economic analyses by reviewers of either profession. As per the case study in the original QHES paper, papers have been assessed as high $(\geq 75 / 100$ points $)$, medium (50-74 points) or low ( $<50$ points) quality.

\section{Data analysis}

For the cost of each activity within the maternity pathway, the range, mean, $\mathrm{SD}$ and relative difference between the minimum and maximum estimates were reported. The distribution of costs was represented graphically on a scatter plot, with data divided by cost source (national guideline, review article and primary research study).

Simulated low-risk and high-risk patients were agreed through consensus of the clinical coauthors with reference to risk stratification guidance produced by NICE, to demonstrate the difference in cost estimates for common exemplar clinical scenarios when applied across the whole maternity pathway. ${ }^{13}$ The planned low-risk pregnant woman was 35 years old and multiparous, having had two previous vaginal births with no medical or obstetric complicating factors. She had an uncomplicated pregnancy and spontaneous vaginal birth, followed by a 6 -hour postnatal discharge. The planned high-risk woman was 42 years old and nulliparous, having conceived with in vitro fertilisation. She develops pre-eclampsia in the 35th gestational week and is induced at 37 weeks' gestation.
She labours with an epidural but requires an emergency caesarean section for fetal distress.

Two post hoc sensitivity analyses were conducted to determine; (1) the extent to which removal of low-quality primary research papers reduced the variability in cost estimates and (2) whether the variation in cost estimates for each named activity was caused by the range of different cost perspectives used. For the first analysis, costs derived from published papers deemed to be of low quality were removed, and the effect on the mean and range costs per activity was described. For the second analysis, cost estimates for each activity included in the primary research articles were presented graphically, stratified by the perspective.

\section{Patient and public involvement}

A lay representative from Guy's and St Thomas' charity (AA) was involved in the early set-up of the DESIGN trial, the economic evaluation of which motivated this review. $\mathrm{He}$ is a coauthor of the DESiGN Trial Team group, who reviewed the final draft manuscript.

\section{Ethical review}

Ethical review is not required in the UK for systematic reviews.

\section{RESULTS}

Of 5081 papers identified in the electronic database search, 3 economic evaluations in relevant NICE guidelines and 10 publications identified from handsearching systematic reviews, 848 were duplicates and 4080 were excluded through screening of the titles and abstracts, leaving 140 full texts for screening. Following exclusion of papers that did not meet the inclusion criteria, 37 papers were included in the final review, including 27 primary research articles, 7 review articles and 3 economic evaluations from NICE guidelines. This is represented diagrammatically in figure 1 .

The characteristics of the included studies are detailed in online supplemental file 3. Of the included primary research studies $(n=27)$, four costed the healthcare activities from the perspective of the local hospital (ie, direct costs of procuring items and paying staff salaries at those hospitals), one costed from the perspective of the commissioner (ie, direct costs of paying the hospital for providing a service), two costed from the indirect societal perspective (ie, the wider costs to society, including work days lost; in one case this includes the NHS perspective) and the remaining studies $(n=20)$ (including guidelines and review articles) costed from the NHS perspective only (ie, directly attributed, nationally agreed costs for procurement, staff salaries and so on).

In quality assessment of the 27 primary research articles, 21 were scored as high quality ( $\geq 75 / 100$ points), ${ }^{14-34}$ 3 as medium quality $(50-74 / 100 \text { points })^{35-37}$ and 3 were scored as low quality (<50/100 points).$^{38-40}$ The detailed 


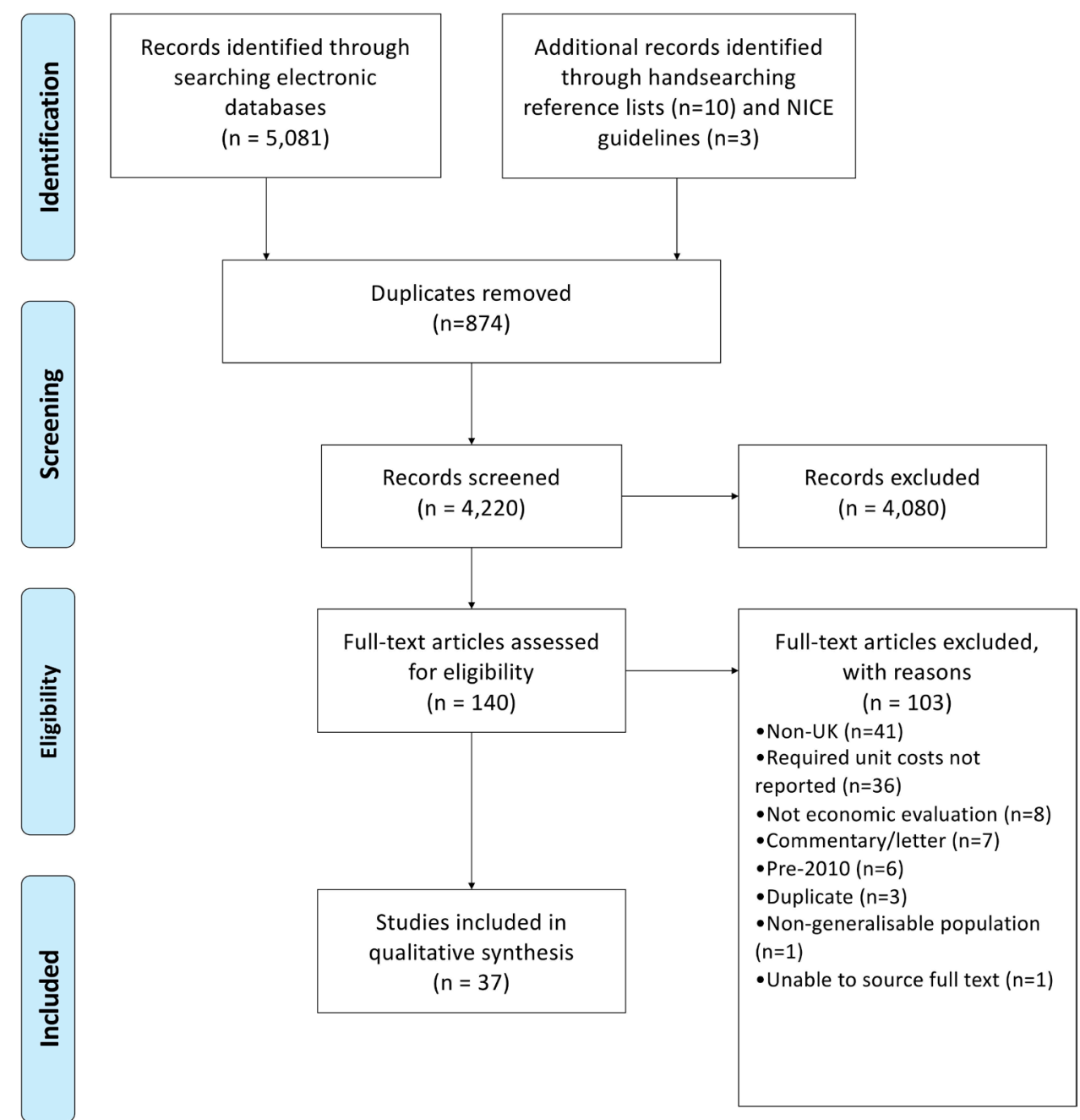

Figure 1 Study selection process. NICE, National Institute for Health and Care Excellence.

QHES evaluations are provided in online supplemental file 4.

Of the primary research articles $(n=27)$, seven estimated activity costs using bottom-up methodologies (ie, individually microcosted each item, for example, drug costs, equipment costs and cost per staff-hour worked), 10 costed activities using national reference costs for NHS diagnosis or procedure codes and staff, 3 used other available literature to cost activities and the remaining 7 studies used a combination of costing methodologies.

The cost estimates from the NICE guidelines, review articles and primary research papers (inflated to 2018/2019 prices) are presented separately for activity items within antenatal, intrapartum and postnatal care within figure 2 and in more detailed and referenced format in online supplemental files 5-7, respectively.

With respect to antenatal care, estimates for $20 \mathrm{~min}$ long antenatal clinic appointments were provided for midwifery-led clinics (range £27.34-£146.25, mean £74.70, 5.3-fold difference) $)^{29} 3436$ 41-43 or consultant obstetrician-led clinics (range £43.36-£312.29, mean £144.15, 7.2-fold difference). 2225293436414244 There were only two cost estimates identified for glucose tolerance tests (range $£ 13.03-£ 26.16$, mean $£ 21.80,2$.0-fold difference). A larger absolute unit cost range was found when estimating the cost for 1 day of an antenatal inpatient admission (range £298.47-£1115.87, mean $£ 546.08$, 3.7-fold difference). ${ }^{1415}$ 18-21 252834 36-38 44-46 Similarly, cost estimates for antenatal scans were variable for both general scans conducted by a sonographer (range $£ 40.67-$ $£ 139.85$, mean £80.86, 3.4-fold difference) $29303536424345-49$ and 'specialist' scans, usually conducted by a fetal medicine consultant (range $£ 77.82-£ 143.65$, mean $£ 116.34$, 1.8-fold difference). 252938

When estimating cost for intrapartum activities, there is wide variation, and the costs for each activity item are generally higher than they are for antenatal or postnatal care. For example, the estimated cost of induction of labour varies between $£ 47.56$ and $£ 805$.42 (mean $£ 450.08$, 16.9-fold difference). ${ }^{171920222841434749-51}$ In some papers, it was clear that this variation follows decisions to cost the induction with or without the cost of staffing and antenatal admission, but this is not always the case. The estimated cost of an emergency caesarean section varies from £1056.44-£4982.21 (mean £3508.93, 4.7-fold difference) ${ }^{1318-222428-30323641424749-51}$; this includes the staffing and bed space required for the intrapartum admission. There are lower estimates that cost the surgery only (£318.78-£1432.71, 4.5-fold difference). ${ }^{15} 173343$ 
A

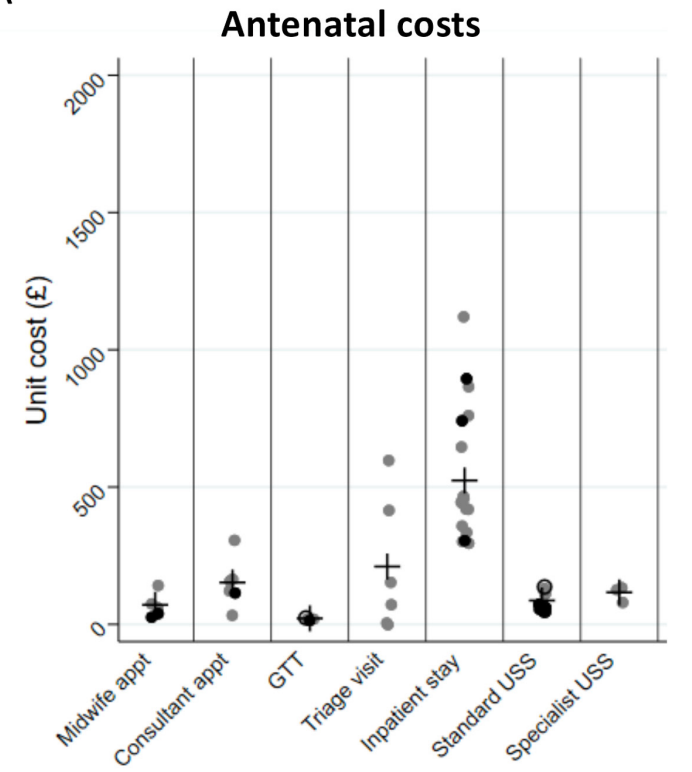

B

\section{Postnatal costs}

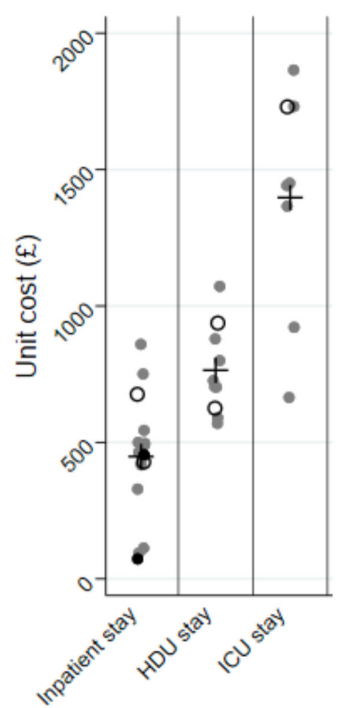

C

Intrapartum costs

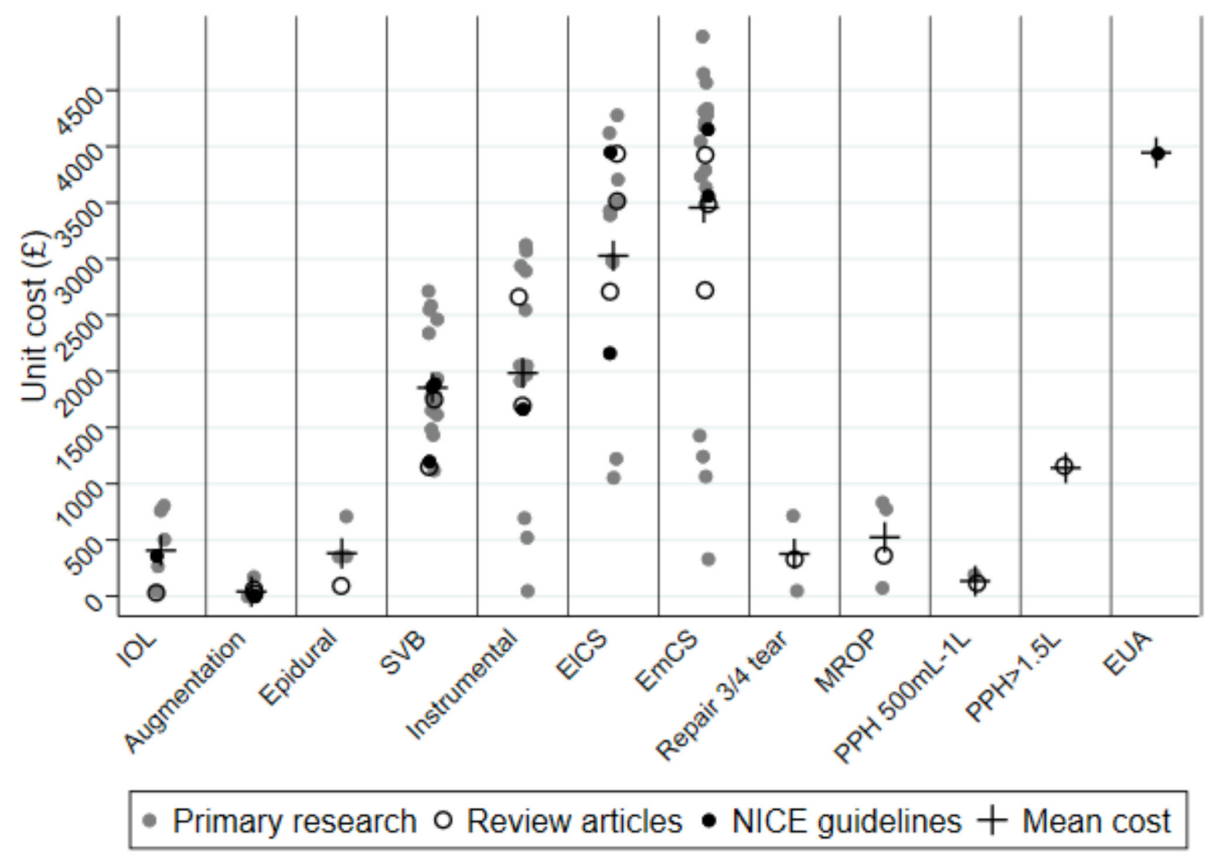

Figure 2 Variation in extracted unit costs for activities within the maternity care pathway. EICS, elective caesarean section; EmCS, emergency caesarean section; EUA, examination under anaesthesia; GTT, glucose tolerance test; HDU, high dependency unit; ICU, intensive care unit; IOL, induction of labour; MROP, manual removal of placenta; NICE, national institute for health and care excellence; PPH, postpartum haemorrhage; SVB, spontaneous vaginal birth; USS, ultrasound scan.

With regards to postnatal care, the inpatient postnatal stay for a healthy woman and baby on a postnatal ward varies between $£ 103.00$ and $£ 870.10$ per day (mean $£ 469.55,8$.4-fold difference). ${ }^{13-1521} 2324272833363940445052$

Through application of cost estimates to the exemplar activities within the care pathway for a low-risk multiparous woman and high-risk nulliparous woman and applying the lowest and highest cost estimates, we have demonstrated the significant effects that these cost variations can have on the estimated cost of care provided to a single woman (tables 2 and 3 ).

Sensitivity analysis with exclusion of the three primary research papers that were assessed as low quality using the QHES instrument resulted in only two cost estimates being removed from the overall results. The mean cost per antenatal admission day changed from £524.11 (SD: $£ 239.07$ ) to £528.51 (SD: £248.24), and the mean cost per day on postnatal ward changed from $£ 471.92$ (SD: 
Table 2 Estimating costs for a low-risk pregnant woman

\begin{tabular}{lll}
\hline $\begin{array}{l}\text { Activity within care } \\
\text { pathway* }\end{array}$ & $\begin{array}{l}\text { Lowest cost } \\
\text { estimate }\end{array}$ & $\begin{array}{l}\text { Highest cost } \\
\text { estimate }\end{array}$ \\
\hline $\begin{array}{l}\text { Antenatal booking } \\
\text { appointment }\end{array}$ & $£ 27.34^{42}$ & $£ 146.25^{34}$ \\
$\begin{array}{l}\text { Two sonography-led } \\
\text { ultrasound scans }\end{array}$ & $2 \times £ 42.24^{46}$ & $2 \times £ 139.85^{48}$ \\
$£ 84.48$ & $£ 279.70$ \\
\hline $\begin{array}{l}\text { Five midwifery-led } \\
\text { antenatal appointments }\end{array}$ & $5 \times £ 27.34^{42}$ & $5 \times £ 146.25^{34}$ \\
One attendance to & $£ 6.56^{37}$ & $£ 415.65^{25}$ \\
maternity triage & & \\
Uncomplicated & $£ 1125.95^{33}$ & $£ 2572.02^{21}$ \\
spontaneous vaginal birth & & $£ 0$ \\
6-hour discharge & $£ 0$ & $£ 4144.87$ \\
\hline Total & $£ 1381.03$ & \\
\hline
\end{tabular}

*The exemplar lower risk pregnant woman was multiparous, aged 35 years, two previous vaginal births and no medical or obstetric complicating factors.

$£ 211.26$ ) to $£ 469.55$ (SD: 219.69). There were no changes to intrapartum costs.

Sensitivity analysis to determine the effect of including a range of cost perspectives on the variation identified between cost estimates for each activity was conducted graphically, and the results are presented in online supplemental file 8 . The majority of costs are derived from economic evaluations that use the perspective of the health service. The figure demonstrates the extent to which variation still exists, even for costs derived from studies conducted from the perspective of the health service. There were too few data points to examine variation across studies conducted from other perspectives.

\section{DISCUSSION \\ Summary of the key findings}

The aim of this report was to overview the current evidence on the costing of resource use within maternity care to inform economic evaluations of maternity interventions. We have reviewed 7 economic evaluations with UK costs applied following a systematic literature review, 3 economic evaluations from UK NICE guidelines and 27 primary research articles that have attributed unit costs to activity within the antenatal, intrapartum and postnatal pathways, specific to the UK context. We have noted wide ranges in published cost estimates, including a 16.9-fold difference between the minimum and maximum cost estimates for induction of labour, despite limiting the search to studies within the last 10 years and inflating costs to 2018/2019 prices.

Table 3 Estimating costs for a higher risk pregnant woman

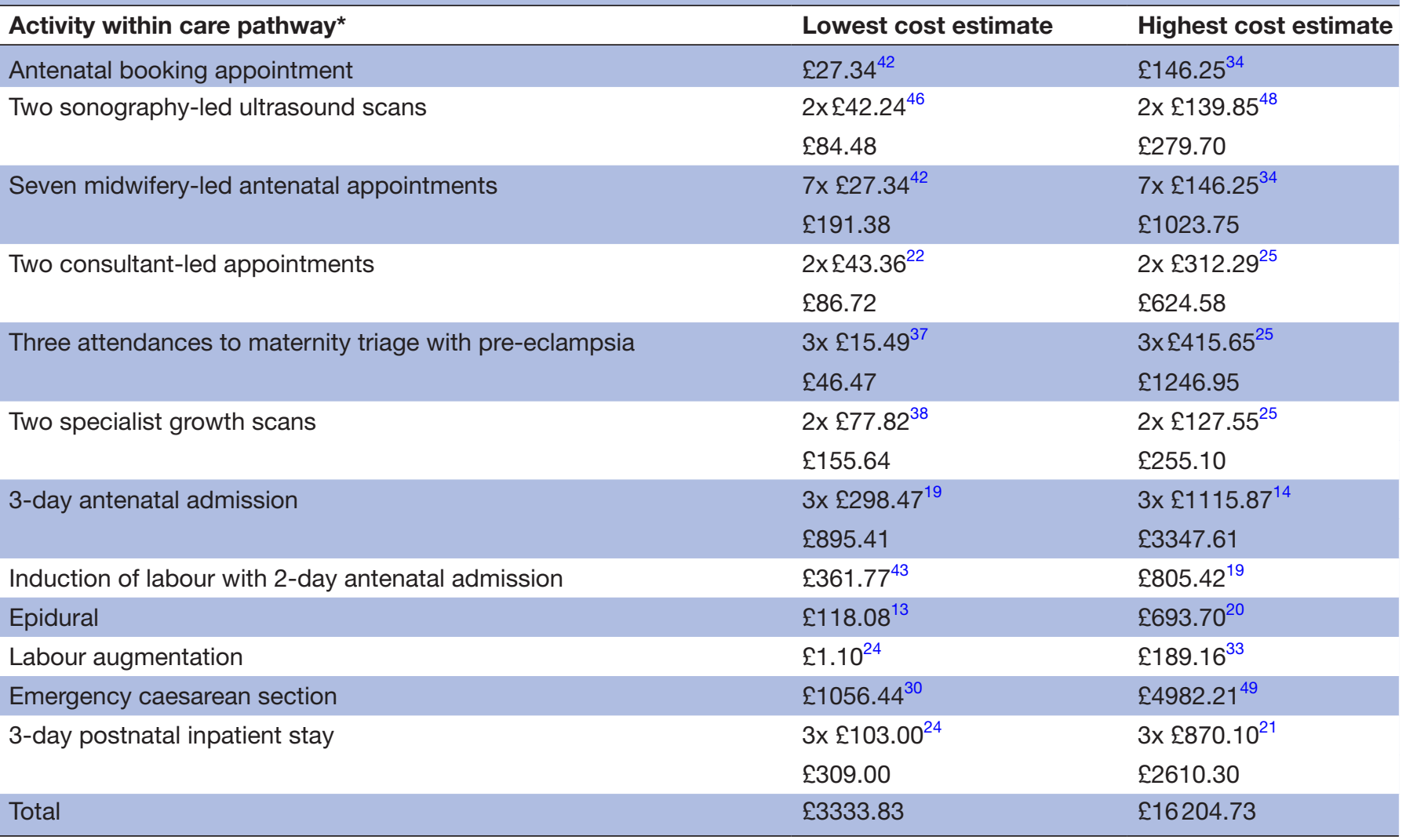

*The exemplar higher risk pregnant woman was nulliparous, aged 42 years and conceived by in vitro fertilisation. Develops pre-eclampsia at 35 weeks. 
For intrapartum costs in particular, the absolute difference between the minimum and maximum costs are greater, because these are usually higher cost interventions. This is likely to have more of an impact on the results of cost-effectiveness evaluations. Even where absolute cost differences are small because the activity itself is relatively inexpensive, for example, cost estimates for the glucose tolerance test (GTT), the relative difference shows that the maximum estimate is twice as high (or more) than the minimum estimate, although a low absolute difference is less likely to impact when estimating the financial impact of new interventions.

While the estimate ranges are tighter for the unit costs supplied in the seven review articles, with the overall estimate tending towards the middle of the range of the primary research articles, this may be accounted for by both the smaller number of studies and that these studies are often based on estimates from the national guidelines and primary research articles. Wide variation also exists within cost estimates supplied by some NICE guidelines, where the same activity (eg, emergency caesarean section) is priced differently by economic evaluations featured in different guidelines. ${ }^{1341} 4750$

\section{Interpretation of study findings and comparison with existing literature}

There are several potential explanations for some of this variation. Variation can reflect different methodology of cost calculation and differing definitions of each activity, for example, the average cost for an inpatient admission varies from cost per day/night to costs estimated for a time-defined (eg, 3 nights) admission (although only costs for a single night are presented in the results). Costs may also vary with changing geographical perspective and varying approaches to clinical practice resource use between localities; it is well established that costs are higher in Southern England, particularly inner-city London. ${ }^{53}$ Methodological quality is another explanation for the variation found in this review, although the estimates changed little after exclusion of papers determined to be of low methodological quality. Poorly applied methods and incomplete reporting make the results of economic evaluations less reliable, less comparable on a consistent like-with-like basis and difficult to interpret. It also introduces additional uncertainty when seeking to transfer evidence on costs to other study contexts.

\section{Implications of this systematic review}

In this paper, we have shown how variation in reported costs can introduce uncertainty into estimates of the overall cost of pregnancy management at different levels of pregnancy risk. This is likely to have important implications where 'bottom-up' costing methodologies are required to support the evaluation of interventions that are expected to change the type and volume of clinical activity that patients are exposed to along the pregnancy care pathway. This will be further magnified in cases where an intervention impacts on comparatively expensive areas of clinical activity, for example, antenatal admissions, method of birth and infant admission to neonatal units.

This review was originally motivated by an economic evaluation of the complex antenatal intervention (Growth Assessment Protocol $)^{54}$ studied within the DEtection of the Small for GestatioNal age Fetus (DESiGN) trial. ${ }^{55}$ DESiGN is a cluster-randomised controlled trial, the primary aim of which is to report on the clinical effectiveness of the GAP programme; secondary outcomes include a process and economic evaluation. GAP aims to improve the rate of antenatal detection of small for gestational age fetuses. ${ }^{54}$ Estimating the financial impact of introducing an intervention into the antenatal pathway was expected to be challenging because of the bundled nature of national reference costs in England. ${ }^{4}$ Our hypothesis specified that the intervention was expected to increase antenatal activities such as clinic appointments or scans and intrapartum activities such as induction of labour. While these changes were expected to incur cost to the hospital, this would not be reflected in the bundled price charged to the commissioner. Itemised costs were therefore required.

As a result of the findings of this review, we have planned for sensitivity analyses to play a central role in the costeffectiveness analysis of the DESiGN trial so that uncertainty in the magnitude of costs linked to key resource use items on cost-effectiveness conclusions can be fully examined and reported. We recommend for other triallists to do the same when conducting economic evaluations in settings where widely agreed itemised costs are not available.

The variation in quoted costs suggests uncertainty around methods to calculate costs. While the included studies have mostly been appraised as having medium or high reporting quality (according to the QHES checklist), it was not always explicit how costs were calculated and exactly what was included in each estimate, for example, length of appointment, salary of healthcare professional used and inclusion of indirect costs. Guidelines on what should be included when calculating the cost of common activities, including how to account for variable staff salaries and indirect costs and recommendations on the appropriate cost perspective to choose (and report) and how to translate costs geographically, would be invaluable in achieving lower variation in published estimates.

An alternative strategy would be publication of a list of nationally agreed itemised costs for use in the economic evaluation of interventions, with guidance on which costs within a range of estimates are more likely to be applicable to specific circumstances. This would facilitate greater consistency in the application of cost data across different evaluations. Such a list was previously available in England (online supplemental file 9) but has since been replaced by a national bundled tariff. ${ }^{56}$

\section{Strengths and limitations}

The strength of this study is in the extensive literature search of four relevant databases and the wide, clinically 
generalisable (within UK maternity care) inclusion criteria. Unit costs extracted from published research articles were compared with one another and with summary costs published as part of national Health Technology Assessments and guidelines. Unit costs extracted from published reports of economic evaluations were inflated to $2018 / 2019$ prices and stratified by quoted cost perspective to ensure comparability.

Due to the lack of comparability in international health systems and maternity reimbursement policies, it was not appropriate to extend the search outside of economic evaluations conducted within the UK. While the specific cost findings are only generalisable to UK maternity care, the overall findings regarding the challenges of estimating the financial impact of interventions using bundled prices, and the risk of cost variation where nationally agreed costs are not available, are relevant to maternity care providers internationally and potentially also to other medical specialities where bundled costs are commonplace.

\section{CONCLUSIONS}

Through this systematic review of economic evaluations within maternity care, we have described variation in costs applied to maternity care activities, even after controlling for study reporting quality and cost perspective. We have outlined the challenges in attributing cost to maternity activities, due to non-standardised activity descriptions and provision of composite 'bundled' cost estimates.

Overall, the level of variation in cost calculations is likely to reflect the uncertainty within the system and must be dealt with by conducting sensitivity analyses of economic evaluations. The development of nationally agreed unit costs for key areas of clinical activity within the pregnancy care pathway would serve to standardise cost-effectiveness evaluations of new interventions within maternity care to be used either for research purposes or national decisions regarding intervention uptake.

\section{Author affiliations}

'Department of Women and Children's Health, King's College London, St Thomas' Hospital, London, UK

${ }^{2}$ Health Improvement: Alcohol, Drugs, Tobacco and Justice Division, Public Health England, London, UK

${ }^{3}$ Department of Obstetrics and Gynaecology, School of Medicine, Pontifícia Universidade Católica do Rio Grande do Sul, Porto Alegre, Brazil

${ }^{4}$ Fetal Medicine Unit, St George's University Hospitals NHS Foundation Trust, London, UK

${ }^{5}$ Molecular \& Clinical Sciences Research Institute, St George's, University of London, Cranmer Terrace, London, UK

${ }^{6}$ Discipline of Obstetrics, Gynaecology \& Neonatology, Westmead Clinical School, Faculty of Medicineand Health, University of Sydney, Sydney, New South Wales, Australia

${ }^{7}$ Health Service and Population Research, King's College London, De Crespigny Park, London, UK

\section{Twitter Sophie Relph @sophie_relph}

Acknowledgements In addition to the named authors of this study, The DESiGN Trial team is composed of the following coinvestigators who conceived the original trial and have supported its ongoing progress: AC (Centre for Pragmatic Global
Health Trials, Institute for Global Health, University College London), KC (Faculty of Health, Social Care and Education, Kingston and St. George's University), AA (Lay representative, Guy's \& St Thomas' Charity), AB (Department of Women and Children's Health, School of Life Course Sciences, Faculty of Life Sciences and Medicine, King's College London), MJ (Department of Surgery and Cancer, Imperial College London), DAL (Population Health Science, Bristol Medical School, University of Bristol), CL (Department of Surgery and Cancer, Imperial College London), NM (UCL Institute for Women's Health, University College London), LMC (Faculty of Medical and Health Sciences, University of Auckland, New Zealand), LP (West Middlesex University Hospital, Chelsea \& Westminster Hospital NHS Foundation Trust), DP (UCL Institute for Women's Health, University College London), AS (Department of Women and Children's Health, School of Life Course Sciences, Faculty of Life Sciences and Medicine, King's College London) and BT (Fetal Medicine Unit, St George's University Hospitals NHS Foundation Trust and Molecular \& Clinical Sciences Research Institute, St George's, University of London).

Contributors SR conducted the literature search, data extraction and wrote the full report. LD and AM extracted data following the literature review. MCV produced the figures for graphical representation of the extracted data. DP, AH, JS and AK are coinvestigators of the DESiGN trial and conceived the health economic evaluation contained within the trial and the need for this review. DP and AH supervised the review, data interpretations and drafting of the report. All authors reviewed the final draft and revised where necessary.

Funding The DESiGN trial has been funded by the Guy's and St Thomas' Charity (MAJ150704), Sands Charity(RG1011/16) and Tommy's charity UK (core support, London centre, grant number N/A). DP was also individually funded by Tommy's charity. JS is an NIHR Senior Investigator and with SR, and AH, are supported by the National Institute for Health Research (NIHR) Applied Research Collaboration (ARC) South London at King's College Hospital NHS Foundation Trust. MCV was supported by a Science Without Borders Fellowship from CAPES, Brazil (BEX: 9571/13-2). AM was funded by a King'slmprovement Science fellowship, funded by Guy's and St Thomas' and Maudsley Charity. The viewsexpressed are those of the authors and not necessarily those of the NIHR, Department of Health andSocial Care or funding charities.

Dissemination declaration The report of this study has been shared with the lay reviewer from Guy's and St Thomas' charity, as a coinvestigator of the study. Further dissemination for patient groups is not applicable.

Competing interests None declared.

Patient consent for publication Not required.

Provenance and peer review Not commissioned; externally peer reviewed.

Data availability statement All data relevant to the study are included in the article or uploaded as supplementary information. All data used in the analysis of results and synthesis of conclusions have been shared in the supplementary material of this paper.

Supplemental material This content has been supplied by the author(s). It has not been vetted by BMJ Publishing Group Limited (BMJ) and may not have been peer-reviewed. Any opinions or recommendations discussed are solely those of the author(s) and are not endorsed by BMJ. BMJ disclaims all liability and responsibility arising from any reliance placed on the content. Where the content includes any translated material, BMJ does not warrant the accuracy and reliability of the translations (including but not limited to local regulations, clinical guidelines, terminology, drug names and drug dosages), and is not responsible for any error and/or omissions arising from translation and adaptation or otherwise.

Open access This is an open access article distributed in accordance with the Creative Commons Attribution Non Commercial (CC BY-NC 4.0) license, which permits others to distribute, remix, adapt, build upon this work non-commercially, and license their derivative works on different terms, provided the original work is properly cited, appropriate credit is given, any changes made indicated, and the use is non-commercial. See: http://creativecommons.org/licenses/by-nc/4.0/.

ORCID iDs

Sophie Relph http://orcid.org/0000-0002-2007-9647

Matias C Vieira http://orcid.org/0000-0002-8076-4275

\section{REFERENCES}

1 National Institute for Health and Care Excellence. NICE technology appraisal guidance. Available: https://www.nice.org.uk/about/what- 
we-do/our-programmes/nice-guidance/nice-technology-appraisalguidance

2 El Alili M, van Dongen JM, Huirne JAF, et al. Reporting and analysis of trial-based cost-effectiveness evaluations in obstetrics and gynaecology. Pharmacoeconomics 2017;35:1007-33.

3 Independent Hospital Pricing Authority. Bundled pricing for maternity care: final report of IHPA and the bundled pricing Advisory group 2017, 2020. Available: https://www.ihpa.gov.au/sites/default/files/ bundled_pricing_for_maternity_care_-_final_report.docx

4 NHS Improvement. National tariff payment system 2017/18 and 2018/19 2018, 2018. Available: https://improvement.nhs.uk/ resources/developing-the-national-tariff/\#past

5 Medicaid and CHIP Payment and Access Commission. Medicaid payment initiatives to improve maternal and birth outcomes 2019, 2020. Available: https://www.macpac.gov/publication/medicaidpayment-initiatives-to-improve-maternal-and-birth-outcomes/

6 NHS England, NHS Improvement. Guidance on the market forces factor: a supporting document for the 2017 to 2019 national tariff payment system, 2016. Available: https://improvement.nhs.uk/ resources/developing-the-national-tariff/\#past

7 The Commonwealth Fund. Bundled-payment models around the world: how they work and what their impact has been 2020, 2020. Available: https://www.commonwealthfund.org/publications/2020/ apr/bundled-payment-models-around-world-how-they-work-theirimpact

8 Fahy M, Doyle O, Denny K, et al. Economics of childbirth. Acta Obstet Gynecol Scand 2013;92:508-16.

9 Public Health England. Guidance: economic evaluation 2018, 2020. Available: https://www.gov.uk/government/publications/evaluationin-health-and-well-being-overview/economic-evaluation

10 Department of Health. Pay \& price series 2015/16 2016, 2018. Available: www.info.doh.gov.uk

11 NHS Improvement. Economic assumptions for 2016/17 to 2020/21, 2016. Available: https://www.gov.uk/government/publications/ economic-assumptions-201617-to-202021/economic-assumptions201617-to-202021

12 Ofman JJ, Sullivan SD, Neumann PJ, et al. Examining the value and quality of health economic analyses: implications of utilizing the QHES. J Manag Care Pharm 2003;9:53-61.

13 National Institute for Health and Care Excellence. Intrapartum care for healthy women and babies. clinical guideline CG 1902014.

14 Petrou S, Taher S, Abangma G, et al. Cost-Effectiveness analysis of prostaglandin E2 gel for the induction of labour at term. BJOG 2011;118:726-34

15 Eddama O, Petrou S, Regier D, et al. Study of progesterone for the prevention of preterm birth in twins (STOPPIT): findings from a trialbased cost-effectiveness analysis. Int J Technol Assess Health Care 2010;26:141-8.

16 Jit M, Cromer D, Baguelin M, et al. The cost-effectiveness of vaccinating pregnant women against seasonal influenza in England and Wales. Vaccine 2010;29:115-22.

17 Round JA, Jacklin P, Fraser RB, et al. Screening for gestational diabetes mellitus: cost-utility of different screening strategies based on a woman's individual risk of disease. Diabetologia 2011;54:256-63.

18 Essex HN, Parrott S, Wu Q, et al. Cost-Effectiveness of nicotine patches for smoking cessation in pregnancy: a placebo randomized controlled trial (SNAP). Nicotine Tob Res 2015;17:636-42.

19 Coomarasamy A, Williams H, Truchanowicz E, et al. PROMISE: first-trimester progesterone therapy in women with a history of unexplained recurrent miscarriages - a randomised, double-blind, placebo-controlled, international multicentre trial and economic evaluation. Health Technol Assess 2016;20:1-92.

20 Lain SJ, Roberts CL, Bond DM, et al. An economic evaluation of planned immediate versus delayed birth for preterm prelabour rupture of membranes: findings from the PPROMT randomised controlled trial. BJOG 2017;124:623-30.

21 Ussher M, Lewis S, Aveyard P, et al. The London exercise and pregnant smokers (leap) trial: a randomised controlled trial of physical activity for smoking cessation in pregnancy with an economic evaluation. Health Technol Assess 2015;19:1-136.

22 Walker KF, Dritsaki M, Bugg G, et al. Labour induction near term for women aged 35 or over: an economic evaluation. BJOG 2017; 124:929-34.

23 van der Nelson HA, Draycott T, Siassakos D, et al. Carbetocin versus oxytocin for prevention of post-partum haemorrhage at caesarean section in the United Kingdom: an economic impact analysis. Eur J Obstet Gynecol Reprod Biol 2017;210:286-91.

24 Bick D, Briley A, Brocklehurst P, et al. A multicentre, randomised controlled trial of position during the late stages of labour in nulliparous women with an epidural: clinical effectiveness and an economic evaluation (BUMPES). Health Technol Assess 2017;21:1-176

25 Duckworth S, Chappell LC, Seed PT, et al. Placental growth factor (PIGF) in women with suspected pre-eclampsia prior to 35 weeks gestation: a budget impact analysis. PLoS One 2016;11:e0164276.

26 Orlovic M, Carter AW, Marti J, et al. Estimating the incidence and the economic burden of third and fourth-degree obstetric tears in the English NHS: an observational study using propensity score matching. BMJ Open 2017;7:e015463.

27 Khan KS, Moore P, Wilson M, et al. A randomised controlled trial and economic evaluation of intraoperative cell salvage during caesarean section in women at risk of haemorrhage: the SALVO (cell salvage in obstetrics) trial. Health Technol Assess 2018;22:1-88.

28 Waugh J, Hooper R, Lamb E, et al. Spot protein-creatinine ratio and spot albumin-creatinine ratio in the assessment of pre-eclampsia: a diagnostic accuracy study with decision-analytic model-based economic evaluation and acceptability analysis. Health Technol Assess 2017;21:1-90.

29 Jones M, Smith M, Lewis S, et al. A dynamic, modifiable model for estimating cost-effectiveness of smoking cessation interventions in pregnancy: application to an RCT of self-help delivered by text message. Addiction 2019;114:353-65.

30 Jacklin PB, Maresh MJ, Patterson CC, et al. A cost-effectiveness comparison of the NICE 2015 and who 2013 diagnostic criteria for women with gestational diabetes with and without risk factors. BMJ Open 2017;7:e016621.

31 Wastlund D, Moraitis AA, Thornton JG, et al. The cost-effectiveness of universal late-pregnancy screening for macrosomia in nulliparous women: a decision analysis. BJOG: Int J Obstet Gy 2019;126:1243-50.

32 Wastlund D, Moraitis AA, Dacey A, et al. Screening for breech presentation using universal late-pregnancy ultrasonography: a prospective cohort study and cost effectiveness analysis. PLoS Med 2019;16:e1002778.

33 Schroeder E, Petrou S, Patel N, et al. Cost effectiveness of alternative planned places of birth in woman at low risk of complications: evidence from the birthplace in England national prospective cohort study. BMJ 2012;344:e2292.

34 Vatish M, Strunz-McKendry T, Hund M, et al. sFIt-1/PIGF ratio test for pre-eclampsia: an economic assessment for the UK. Ultrasound Obstet Gynecol 2016;48:765-71.

35 Carolan-Rees G, Ray AF. The ScanTrainer obstetrics and gynaecology ultrasound virtual reality training simulator: a cost model to determine the cost viability of replacing clinical training with simulation training. Ultrasound 2015;23:110-5.

36 Campbell HE, Kurinczuk JJ, Heazell A, et al. Healthcare and wider societal implications of stillbirth: a population-based cost-of-illness study. BJOG 2018;125:108-17.

37 Xydopoulos G, Perry H, Sheehan E, et al. Home blood-pressure monitoring in a hypertensive pregnant population: cost-minimization study. Ultrasound Obstet Gynecol 2019;53:496-502.

38 Parisaei M, Currie J, O'Gorman N, et al. Implementation of foetal fibronectin testing: Admissions, maternal interventions and costs at 1 year. J Obstet Gynaecol 2016;36:888-92.

39 Bowers J, Cheyne H. Reducing the length of postnatal hospital stay: implications for cost and quality of care. BMC Health Serv Res 2016;16:16

40 Luni Y, Borakati A, Matah A, et al. A prospective cohort study evaluating the cost-effectiveness of carbetocin for prevention of postpartum haemorrhage in caesarean sections. J Obstet Gynaecol 2017;37:601-4

41 National Institute for Health and Care Excellence. Inducing labour, 2008. Available: https://www.nice.org.uk/guidance/cg70/resources/ inducing-labour-pdf-975621704389

42 Mistry $\mathrm{H}$, Heazell AEP, Vincent $\mathrm{O}$, et al. A structured review and exploration of the healthcare costs associated with stillbirth and a subsequent pregnancy in England and Wales. BMC Pregnancy Childbirth 2013;13:236.

43 Farrar D, Simmonds M, Griffin S, et al. The identification and treatment of women with hyperglycaemia in pregnancy: an analysis of individual participant data, systematic reviews, meta-analyses and an economic evaluation. Health Technol Assess 2016;20:1-348.

44 Thomas CM, Cameron S. Can we reduce costs and prevent more unintended pregnancies? A cost of illness and cost-effectiveness study comparing two methods of EHC. BMJ Open 2013;3:e003815.

45 Deshpande SN, van Asselt ADI, Tomini F, et al. Rapid fetal fibronectin testing to predict preterm birth in women with symptoms of premature labour: a systematic review and cost analysis. Health Technol Assess 2013;17:1-138

46 O'Donnell A, McParlin C, Robson SC, et al. Treatments for hyperemesis gravidarum and nausea and vomiting in pregnancy: a 
systematic review and economic assessment. Health Technol Assess 2016;20:1-268.

47 National Institute for Health and Clinical Excellence. Antenatal care for uncomplicated pregnancies. NICE clinical guidelines 2008.

48 National Institute for Health and Care Excellence. Diabetes in pregnancy: management from preconception to the postnatal period 2015.

49 Wastlund D, Moraitis AA, Thornton JG, et al. The cost-effectiveness of universal late-pregnancy screening for macrosomia in nulliparous women: a decision analysis. BJOG 2019;126:1243-50.

50 National Institute for Health and Care Excellence. Hypertension in pregnancy: diagnosis and management, 2011: 1-51.

51 Alfirevic Z, Keeney E, Dowswell T, et al. Which method is best for the induction of labour? A systematic review, network metaanalysis and cost-effectiveness analysis. Health Technol Assess 2016;20:1-584.
52 Gallos I, Williams H, Price M, et al. Uterotonic drugs to prevent postpartum haemorrhage: a network meta-analysis. Health Technol Assess 2019;23:1-356.

53 England N, Improvement N. Market forces factor review and proposed updates: NHS 2018.

54 Clifford S, Giddings S, South M, et al. The growth assessment protocol: a national programme to improve patient safety in maternity care. MIDIRS Midwifery Digest 2013;23:516-23.

55 Vieira MC, Relph S, Copas A, et al. The design trial (detection of small for gestational age neonate), evaluating the effect of the growth assessment protocol (GAP): study protocol for a randomised controlled trial. Trials 2019;20:154.

56 Department of Health. Reference costs 2015-16 2016, 2018.

Available: https://assets.publishing.service.gov.uk/government/ uploads/system/uploads/attachment data/file/577083/Reference Costs_2015-16.pdf 